Descargo de responsabilidad: las opiniones expresadas en los manuscritos son responsabilidad exclusiva de los autores. No necesariamente reflejan las opiniones de la editorial ni la de sus miembros.

LENRO

\title{
Implicaciones neurocognitivas por el uso de dispositivos digitales en los adolescentes
}

\section{Neurocognitive implications of the use of digital devices in adolescents}

Recibido: $12 / 10 / 2021$

Publicado: 20/01/2022

\section{Filiación institucional de los autores}

Maestría en Neurociencia con énfasis en Neurocognición

Universidad de San Carlos de Guatemala
Liliana Elizabeth Monroy Díaz monroydiaz29@gmail.com

https://orcid.org/0000-0002-0038-5209

Jim Roberto Castillo Mendoza

jimctof@gmail.com

https://orcid.org/0000-0001-5473-3996

Dick Randolfo Guillén Dardon

dr.dick.randolfo.guillen@gmail.com https://orcid.org/0000-0002-9453-1392

Alex Ovidio Hernández Aldana psicoherald@gmail.com https://orcid.org/0000-0002-3434-3189

Edwin Adolfo Nájera González centrodolorcronico@gmail.com https://orcid.org/0000-0002-1547-6103

\section{Referencia}

Monroy Díaz, L. E., Castillo Mendoza, J. R., Guillén Dardon, D. R., Hernández Aldana, A. O., \& Nájera González, E. A. (2022). Implicaciones neurocognitivas por el uso de dispositivos digitales en los adolescentes. Revista Académica Sociedad Del Conocimiento Cunzac, 2(1), 17-24. DOI: https://doi.org/10.46780/sociedadcunzac.v2i1.8

\section{Resumen}

OBJETIVO: deducir las implicaciones que tiene el uso de dispositivos digitales en los adolescentes. MÉTODO: se realizó un estudio descriptivo transversal con 146 adolescentes estudiantes de 12 a 17 años de edad, en el municipio de Guastatoya, departamento El Progreso. RESULTADOS: el rango etario con mayor frecuencia fue de 16 años con un $34 \%$, $(\mathrm{N}=50)$ en cuanto al género que predominó fue el femenino con un $63.7 \%(\mathrm{~N}=93)$. Los adolescentes respondieron que utilizan dispositivos digitales para la conexión de clases virtuales en un $62.6 \%$ $(\mathrm{N}=92)$ y la herramienta que más utilizan es el teléfono celular con un $55.1 \%$. $(\mathrm{N}=80)$. Además, la inversión de tiempo que están dando a diario es de cuatro a seis horas haciendo un total de $24.5 \%(\mathrm{~N}=36)$ y en un máximo de 12 o más horas un 19\% ( $\mathrm{N}=28)$ de adolescentes. CONCLUSIÓN: en la medición del impacto se reflejan tres niveles educativo, social y actitudinal. El primero identifica un $32 \%(\mathrm{~N}=47)$ En el nivel social se logró identificar que las relaciones familiares fueron afectadas desde el punto que generaron impacto en un $42.2 \%(\mathrm{~N}=62)$. El nivel actitudinal o personal se midió con un $49 \%(\mathrm{~N}=72)$ de casos. 


\section{Palabras clave}

adolescentes, neurocognición, dispositivos digitales

\section{Abstract}

OBJECTIVE: to deduce the implications of the use of digital devices in adolescents. METHOD: a cross-sectional descriptive study was carried out with 146 adolescent students between 12 and 17 years of age, in the municipality of Guastatoya, department of El Progreso. RESULTS: the age range with the highest frequency was 16 years with $34 \%,(N=50)$ as for the gender that predominated was the female with $63.7 \%(N=93)$. Adolescents responded that $62.6 \%(\mathrm{~N}=92)$ use digital devices to connect virtual classes and the tool they use the most is the cell phone with $55.1 \%$. ( $(\mathrm{N}=80)$. In addition, the investment of time that they are giving daily is four to six hours making a total of $24.5 \%(\mathrm{~N}=36)$ and in a maximum of 12 or more hours $19 \%(\mathrm{~N}=28)$ of adolescents. CONCLUSION: the impact measurement reflects three educational, social and attitudinal levels. The first identifies $32 \%(\mathrm{~N}=47)$ At the social level, it was possible to identify that family relationships were affected from the point that they generated an impact in $42.2 \%(N=62)$. The attitudinal or personal level was measured with $49 \%(N=72)$ of cases.

\section{Keywords}

adolescents, neurocognition, digital devices

\section{Introducción}

Los datos históricos de la tecnología engranan procesos no solo de información, sino también de la comunicación humana, mostrando efectos tanto positivos como negativos. (Buckingham, 2002). Asumiendo que la adolescencia es una etapa de desarrollo intelectual (según Piaget), que a nivel demográfico representan un grupo de riesgo en su afán de experimentar nuevas experiencias. La categorización de pensamientos meramente abstractos; la lógica, el razonamiento deductivo-inductivo y viceversa; aún son factores que dependerán de la estimulación ambiental y maduración cerebral (Echeburúa \& De Corral, 2010).

Aunado a lo anterior; los adolescentes tienden a pasar de un estado de seguridad social a un estado de vulnerabilidad, esto a raíz de la constante divulgación que los medios tecnológicos parecen dejar día con día una visión de indispensables para cualquier individuo, incluso pasan a generar un estado de dependencia a las llamadas ciberadicciones (Terán, 2019). Un aspecto positivo hace hincapié que los medios digitales o el uso de internet generan al adolescente una demanda de nuevas habilidades para el manejo del espacio digital.

Ante estos nuevos mecanismos, existen aspectos de riesgo que pueden estar influenciando la modificación de conductas; perspectivas de vida; trastornos psicológicos que pueden incluir cambios del estado cognitivo, la regulación emocional o el comportamiento, pérdida del interés por las dinámicas sociales presenciales. Ante esto, es de suma importancia evidenciar cómo la población de adolescentes está respondiendo al uso constante de medios digitales; a lo que puede sumarse los cambios efectuados por la actual crisis sanitaria, los confinamientos y perdida de socialización entre pares; situación que está impactando en el aprendizaje, en lo social y actitudinal de esta población. Por lo que el presente estudio es parte de las investiga- 
ciones del proceso de maestría del Centro Universitario del Zacapa de la Universidad de San Carlos de Guatemala.

\section{Material y métodos}

El estudio es descriptivo de corte transversal. El instrumento que se utilizó fue el de escalas de actitudes de Likert adecuado a un formulario de Google; se aplicó a 146 adolescentes de un centro educativo privado del área urbana del municipio de Guastatoya, departamento de El Progreso. Las variables de investigación son las siguientes: Independiente: dispositivos digitales, Dependiente: adolescentes Moderadora: implicaciones neurocognitivas.

\section{Resultados}

Tabla 1. Características sociodemográficas

\begin{tabular}{lcc}
\hline Características sociodemográficas & Cantidad & $\%$ \\
\hline Rango etario & 50 & $34 \%$ \\
\hline 16 & 40 & $27.2 \%$ \\
\hline 15 & 24 & $16.3 \%$ \\
\hline 17 & 16 & $10.9 \%$ \\
\hline 13 & 10 & $6.8 \%$ \\
\hline 12 & 3 & $2 \%$ \\
\hline Sexo & & \\
\hline Femenino & 93 & $63.7 \%$ \\
\hline Masculino & 53 & $36.3 \%$ \\
\hline Tiempo de utilización de dispositivo digital & & \\
\hline a 6 horas & 36 & $24.65 \%$ \\
\hline a 9 horas & 31 & $21.23 \%$ \\
\hline Mayor de 12 horas & 28 & $19.18 \%$ \\
\hline a 3 horas & 25 & $17.12 \%$ \\
\hline 10 a 12 horas & 23 & $15.75 \%$ \\
\hline No hay horarioexacto & 4 & $2.74 \%$ \\
\hline Actividades del uso de dispositivo digital & & \\
\hline Conexión a clases virtuales & 116 & $78.9 \%$ \\
\hline Tareas escolares & 106 & $72.1 \%$ \\
\hline Redes sociales & 92 & $62.6 \%$ \\
\hline Escuchar música & 82 & $55.8 \%$ \\
\hline Video Juegos & 63 & $42.9 \%$ \\
\hline
\end{tabular}

Fuente: elaboración propia con base a la boleta de recolección de datos. 
Nota: La tabla muestra las características sociodemográficas de la investigación, en dichos resultados identificados por orden de frecuencia, se detalla el rango etario; de acuerdo al género se observa la predominancia del sexo femenino con 93 casos que corresponden a $63.7 \%$ y el masculino con 53 casos reflejando al 36.3\%. Conforme al tiempo de utilización del dispositivo digital, este se midió bajo el rango expresado en horas: 1) De cuatro a seis horas con 36 casos $(52.5 \%)$, 2) De siete a nueve horas, 31 casos (21.1\%) 3) Y de 10 a 12 horas, 23 casos (15.6\%).

Según las actividades que realizan los adolescentes mediante el uso de dispositivos digitales encontramos; 1) La conexión a clases virtuales con 116 casos, representando el $78.9 \%$ de la población total encuestada, 2) Las tareas escolares con 106 casos correspondientes al $72.1 \%$, 3) El uso de las redes sociales con 92 casos correspondientes al 62.6\%,4) Escuchar música con 82 casos correspondientes al $55.8 \%$ y 5 ) El uso de videojuegos con 63 casos que corresponden al $42.9 \%$.

Tabla 2. Implicaciones del uso de dispositivos digitales.

\begin{tabular}{lll}
\hline \multicolumn{1}{c}{ Implicaciones } & Cantidad & \\
\hline $\begin{array}{l}\text { Alteraciones del } \\
\text { comportamiento }\end{array}$ & & \\
\hline Hábitos y costumbres & 44 & $29.9 \%$ \\
\hline Estados de ánimo & 37 & $25.2 \%$ \\
\hline Comportamiento diario & 32 & $21.8 \%$ \\
\hline Todos los anteriores & 25 & $17 \%$ \\
\hline Otros & 9 & $6.1 \%$ \\
\hline Alteraciones cognitivas & & \\
\hline Dificultad para conciliar el & 37 & $25.34 \%$ \\
sueño & & \\
\hline Deseos de privacidad & 37 & $25.34 \%$ \\
\hline Apego familiar & 24 & $16.44 \%$ \\
\hline Pertenencia social & 24 & $16.44 \%$ \\
\hline Otros & 11 & $7.53 \%$ \\
\hline Ansiedad & 8 & $5.48 \%$ \\
\hline Disminución del apetito & 6 & $4.11 \%$ \\
\hline
\end{tabular}

Fuente: elaboración propia con base a la boleta de recolección de datos.

La tabla muestra las alteraciones del comportamiento encontradas en la población estudiada, detallados de la siguiente forma: 1) Los hábitos y costumbres reflejado con 44 casos correspondiente al $29.9 \%$ siendo este el de más alta incidencia, 2) Seguido de los estados de ánimo 
con 37 casos que corresponden al 25.2\%. 3) Continuando con el comportamiento diario, con 32 casos que nos dan el $21.8 \%$. 4) La categoría denominada Todos los Anteriores, fue respondida por 25 casos correspondientes al $17 \%$ y Otros con 9 casos que reflejan $6.1 \%$.

En esta misma tabla también fueron evidenciadas las alteraciones cognitivas, siendo la más frecuente 1) La dificultad para conciliar el sueño con 37 casos de corresponden al 54\%, 2) El deseos de privacidad con 37 casos que corresponden al 54\%, 3) El apego familiar con 24 casos que reflejan el 35\% 4) La pertenencia social con 24 casos con el 35\%, 5) Otros con 11 casos, correspondiente al 16\%, 6) Ansiedad con ocho casos reflejado en el $11.6 \%$ 7) Y disminución del apetito con seis casos que reflejan del $8.76 \%$.

Tabla 3. Medición del impacto de los dispositivos digitales mediante escala de Likert.

\begin{tabular}{lll}
\hline Nivel de impacto negativo & Cantidad & \\
\hline Educativo & & \\
\hline De acuerdo & 47 & $21.8 \%$ \\
\hline Desacuerdo & 32 & $18.4 \%$ \\
\hline No aplica & 27 & $17.7 \%$ \\
\hline Totalmente de acuerdo & 26 & $10.2 \%$ \\
\hline Totalmente en desacuerdo & 15 & \\
\hline Social & & $42.2 \%$ \\
\hline De acuerdo & 62 & $28.6 \%$ \\
\hline Desacuerdo & 42 & $18.4 \%$ \\
\hline Totalmente de acuerdo & 27 & $10.9 \%$ \\
\hline Totalmente desacuerdo & 16 & \\
\hline Actitudinal & & $49 \%$ \\
\hline De acuerdo & 72 & $34.7 \%$ \\
\hline Totalmente de acuerdo & 51 & $8.8 \%$ \\
\hline En desacuerdo & 13 & $7.5 \%$ \\
\hline Totalmente en desacuerdo & 11 & \\
\hline
\end{tabular}

Fuente: elaboración propia con base a la boleta de recolección de datos.

La tabla actual, mide el nivel de impacto de los dispositivos digitales, reflejándose en tres niveles, siendo estos: Educativo, Social y Actitudinal. El nivel educativo identifica que la opción 1) De acuerdo existen 47 casos reflejados en el $32 \%$, 2) Y en desacuerdo 32 casos correspondientes al $21.8 \%, 3$ ) En la opción No Aplica hay 27 casos que reflejan al $18.4 \%$, 4) En Totalmente de acuerdo, 26 casos respondieron identificados con el $17.7 \%, 5$ ) y por último en Totalmente en desacuerdo 15 casos con un $10.2 \%$. 
En el nivel social nos muestra que 1) Las relaciones familiares fueron afectadas en 62 casos que respondieron De acuerdo, correspondiente al 42.2\%, 2) Y En desacuerdo con 42 casos que conforman el $28.6 \%$, 3) La opción Totalmente de acuerdo con 27 casos correspondientes al $18.4 \%$ y 4 ) Totalmente en desacuerdo con 16 casos correspondiente al $10.9 \%$.

Desde el nivel actitudinal o personal la respuesta 1) De acuerdo fue respondida por 72 casos correspondientes al 49\%, 2) Totalmente de acuerdo con 51 casos conformando el 34.7\%, 3) En desacuerdo con 13 casos que corresponden al 8.8\%,4) Y totalmente en desacuerdo 11 casos identificándose con el $7.5 \%$

\section{Discusión}

En la actualidad Guatemala como otros países han venido adaptando nuevas metodologías en el contexto tecnológico; esto ha implicado que la población más joven demande el uso de ciertos artefactos digitales, bien sea para uso educativo, laboral o recreativo, situación que hace referencia a nivel mundial.

Múltiples autores han reportado cambios en la cantidad del tiempo que el adolescente está teniendo en relación a los medios digitales, especialmente en redes sociales. Ejemplo de ello se exponen datos de una primera encuesta realizada en Rusia, al inicio de la época de los 90, la mayoría de adolescentes invertía el tiempo en relacionarse con amigos y casi nadie en interacción con la computadora (Barquero \& Calderón 2016).

Para el año 2000 hubo un cambio radical y eso modificó el porcentaje de los adolescentes que consideraban la interacción con la computadora como una actividad favorita, pasó de menos $2 \%$ a $25 \%$. (Sobkin y Evstigneeva 2004). Dicho cambio en el uso de dispositivos digitales en adolescentes es significativo y en constante aumento; pues con la crisis sanitaria a nivel mundial aceleró y se expandió el uso de los mismos, por lo que dichos recursos continuaran siendo parte de la vida cotidiana de infantes y adolescentes. UNICEF (2017), publicó un informe titulado "Estado mundial de la infancia, Niños en un mundo digital" en dicho informe se hace referencia que los teléfonos inteligentes están alimentando una "cultura del dormitorio", y para muchos niños o adolescentes el acceso en línea es cada vez más personal, tiene un carácter más privado y está menos supervisado.

En comparación con los resultados obtenidos en el estudio existe un cambio de hábitos y costumbres de un $29 \%$ y alteraciones emocionales y del estado ánimo en un $17 \%$, parte de la muestra poblacional manifiesta sentir disgusto al no contar con privacidad y hacer uso de dispositivos digitales siendo un $26.5 \%$. En este caso, ambos estudios han demostrado que en efecto los adolescentes forman parte de un gran número de usuarios en la red.

Asimismo se reconoce que el impacto de ciertos medios tecnológicos (redes sociales, plataformas educativas, video juegos y más); está causando un impacto a nivel educativo (problemas de atención, concentración y asimilación de la información), social (menor socialización con pares y familia, perdida de los espacios de convivencia social así como de habilidades de 
interacción social) y actitudinales (una predisposición desfavorable al uso de horarios regulados, poco control ante los cambios emocionales, cambios significativos en el comportamiento, entre otros.

\section{Referencias}

Barquero Madrigal, A. y Calderón Contreras, F. (2016). Influencia de las nuevas tecnologías en el desarrollo adolescente y posibles reajustes. Cúpulas, 11-25. https://www.binasss.sa.cr/ bibliotecas/bhp/cupula/v30n2/art02.pdf

Buckingham, D. (2002). Crecer en la era de los medios electrónicos, https://dialnet.unirioja.es/ servlet/articulo?codigo $=7152361$

Echeburúa \& De Corral. (2010). Adicción a las nuevas tecnologías y la as redes sociales en jóvenes: un nuevo reto. adicciones, (22) 2. 91-95.

DOI: https://doi.org/10.20882/adicciones.196

Fondo de las Naciones Unidas para la infancia. UNICEF (Diciembre 2017). El estado mundial de la infancia 2017: Niños en un mundio digital https://www.unicef.org/es/informes/El-Estado-Mundial-de-la-Infancia-2017

Sobkin, V. y Evstigneeva, I. (2004). Actitudes de los estudiantes hacia los nuevos juegos de la computadora (capítulo 4). Educación y Sociedad 10-38 https://eric.ed.gov/?q=sobkin+y+evstigneeva+2004\&id=EJ748432

Terán Prieto, A. (2019). Ciberadicciones. Adicciones a las nuevas tecnologías (NTIC). Seminario: Ciberadicciones (págs. 131-141). Madrid: Lúa Ediciones 3.0. https://www.aepap.org/ sites/default/files/pags._131-142_ciberadicciones.pdf

\section{Sobre los autores}

Grupo de investigadores de la maestría en Neurociencias con énfasis en Neurocognición del Centro Universitario de Zacapa.

\section{Financiamiento de la investigación}

Con recursos propios de los investigadores

\section{Declaración de intereses}

Declaran no tener ningún conflicto de intereses, que puedan haber influido en los resultados obtenidos o las interpretaciones propuestas. 


\section{Declaración de consentimiento informado}

El estudio se realizó respetando el Código de ética y buenas prácticas editoriales de publicación.

\section{Derechos de uso}

Copyright@ 2022 por Liliana Elizabeth Monroy Díaz, Jim Roberto Castillo Mendoza, Dick Randolfo Guillén Dardon, Alex Ovidio Hernández Aldana, Edwin Adolfo Nájera González.

Este texto está protegido por una licencia Creative Commons 4.0

Este texto está protegido por la Licencia Creative Commons Atribución 4.0 Internacional.

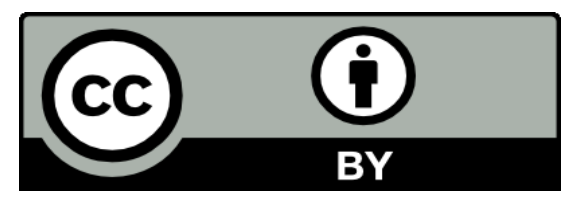

Este texto está protegido por una licencia

Creative Commons 4.0.

Es libre para compartir, copiar y redistribuir el material en cualquier medio o formato y adaptar el documento, remezclar, transformar y crear a partir del material para cualquier propósito, incluso comercialmente, siempre que cumpla la condición de atribución: debe reconocer el crédito de una obra de manera adecuada, proporcionar un enlace a la licencia, e indicar si se han realizado cambios. Puede hacerlo en cualquier forma razonable, pero no de forma tal que sugiera que tiene el apoyo del licenciante o lo recibe por el uso que hace. 